\title{
Smart Real-Time Traffic optimization system
}

\author{
Ramanarayanan $\mathbf{S}^{1}$, Udaya Prakash ${ }^{1}$, Uma Maheshwaran ${ }^{2}$,Velappa Ganapathy ${ }^{3}$, Sundara \\ Kanchana.J ${ }^{4}$
}

\begin{abstract}
Today's traffic situations in busy cities require a smarter traffic signal optimization methodology to regulate the traffic in an orderly manner. Traffic signals are set to a fixed time limit in most of the road junctions. This leads to a dead lock in many junctions because they do not recognize the density of traffic in each road. People want to reach their destinations as quickly as possible. Our proposed Smart Real-Time Traffic Optimization System (SRTOS) provides optimization of traffic signals based on real time Google traffic data. In Google maps, traffic data is indicated through different colors (dark red, red, yellow, orange and green). These colors are processed to find out the traffic around a particular traffic junction and preference is given to the road that has a maximum traffic in the traffic junction, thereby easing out the congestion in that junction. Following similar patterns of activities for all other traffic junctions, wherever possible, will be helpful to drastically ease the flow of traffic patterns of a particular city. This is to fulfill the objective of smoother traffic flow without making people to wait and waste their valuable time.
\end{abstract}

Key Words: Congestion,Google maps, Optimization, Signal, Traffic

\section{INTRODUCTION}

We live in a world where time plays a vital role in everyone's life. Going by the conventional way, many times some unwanted situations might occur where green signal is set to roads having barely any vehicular movement and red is allocated to roads having heavy incoming traffic. This applies to both a morning scenario where people travel from homes to offices and also to an evening scenario where the exact opposite happens. In any such scenarios, extended wait times in traffic signals add up to the wastage of time. People end up spending more time in travel than they intend to. Traffic waiting time also has an adverse effect on the emotional and mental health of people. Optimization of the traffic signals would reduce these issues to a greater extent. Optimization includes removal of allocating equal amount of time for red, green and yellow for each road meeting at a traffic junction and allocating unique or specific times for each of the red, green and yellow signals. The allotment is purely based on the traffic density of each of the roads at a particular instant at that junction. Currently existing optimization solutions prove to be inefficient and quiet

Revised Manuscript Received on July 05, 2019.

Ramanarayanan $\mathbf{S}^{\mathbf{1}^{*} \text { * }}$ Student, Computer Science and Engg.,SSN College of Engg. , College , Kalavakkam- 603110 ,Chennai , Tamil Nadu

Udaya Prakash ${ }^{11}$ Student, Information Technology, SSN College of Engg., College, Kalavakkam- 603110, Chennai , Tamil Nadu , India

Uma Maheshwaran ${ }^{2}$ Student, Information Technology, SSN College of Engg. , College , Kalavakkam- 603110, Chennai, Tamil Nadu , India Velappa Ganapathy ${ }^{3}$ Professor, SRMIST, Kattankulathur,Chennai603110,Tamilnadu, India

Sundara Kanchana. ${ }^{4}$ Academic Administrator, SRMIST,

Kattankulathur,Chennai-603110,Tamilnadu, India expensive. They either use hardware solutions like installing CCTV cameras, thermal sensors etc., which are financially

inconceivable in current situations in developing countries like India. Cheaper solutions usually end up being inefficient and counterproductive. For example, SURTRAC (Smart Urban Traffic Control) proposed by CMU uses sensors to detect flow and density of traffic.

\section{A. METHODOLOGY}

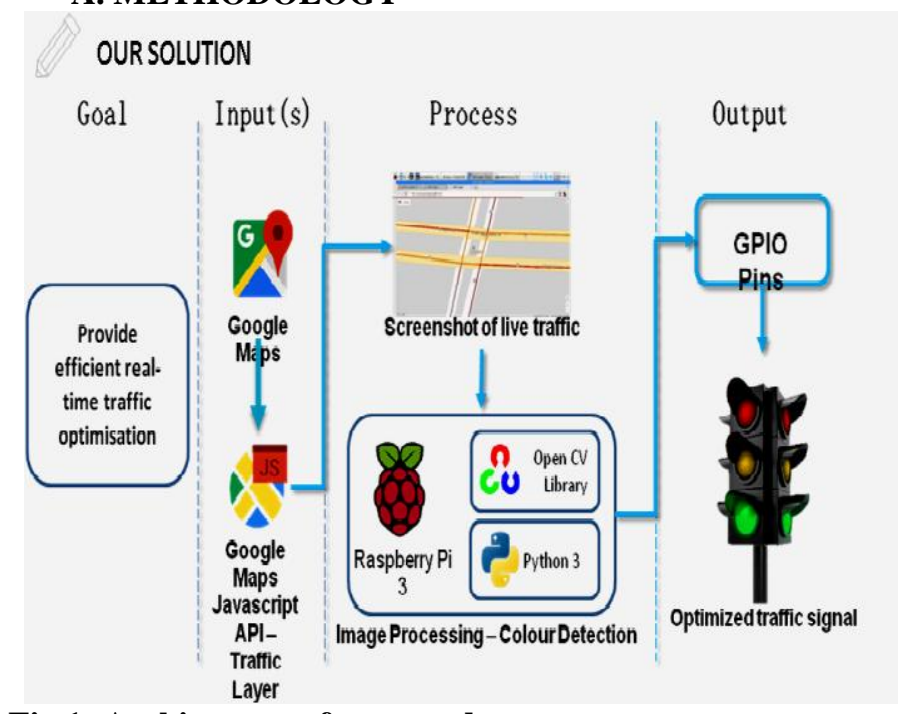

Fig 1: Architecture of proposed system

In Fig 1, the map of a location with a zoomed-in view of a traffic signal only is loaded using the JavaScript API obtained from Google maps, following which, a screenshot of the loaded html file is taken. Using these images, high, medium and low traffic roads around that particular signal are identified using image processing done with Python3 alongside OpenCV library.

The signals are then prioritized by optimizing the green time of a signal according to the image processed traffic data. Raspbian OS is used for operating the Raspberry Pi. If Etcher is not used, there is a need to unzip the .zip downloads to obtain the image file (.img) to write to the inserted SD card. Python is a general purpose programming language which is easy to use and compatible with and hence we have used Python 3 and Raspberry Pi.

To write the image file to an SD card, Etcher is downloaded and installed following which an SD card reader is connected to the SD card inside the Raspberry Pi. Etcher is then opened and the .img or .zip file which is to be opened is selected. Then, the SD card to which the image has been written is selected. Selections are reviewed finally and 'Flash!' is clicked to begin writing to the specified SD card.

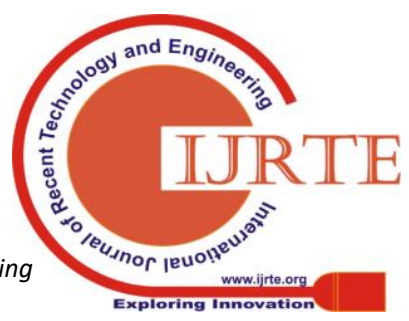


OpenCV-Python is a highly optimized library for numerical operations with a MATLAB-style syntax and this is installed for processing the image that has been written to the SD card previously. OpenCV is a library used for image processing based on many parameters. Screenshots of the webpage is taken for image processing at frequent intervals of time. Initially we had tried by taking screen shots using shell script but when we implemented it, we found that the Python program restarts every time after the screenshot is taken.

\section{B. EXPERIMENTAL SETUP}

We have selected one particular road junction located at ECHANGADU near Chennai, as shown in Fig. 2 for our testing purposes. The steps discussed above are followed in optimizing the traffic flow at the selected junction.

\section{Fig 2 : A Road Junction at EACHANGADU.}

Every time the program is started, a HTML file opens up on the browser with the zoomed-in view of only the signal and the roads around it. This page is refreshed periodically

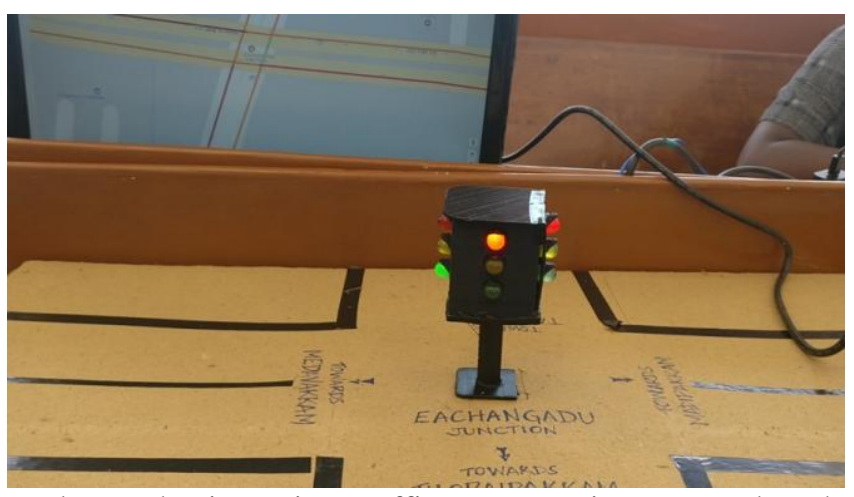

to detect the incoming traffic at every instant so that the Raspberry Pi can process accordingly. Screenshots of this HTML page is taken every time the page is refreshed and is saved as an image and sent for processing. Initially when the program starts, there is a red signal to each of the incoming roads.

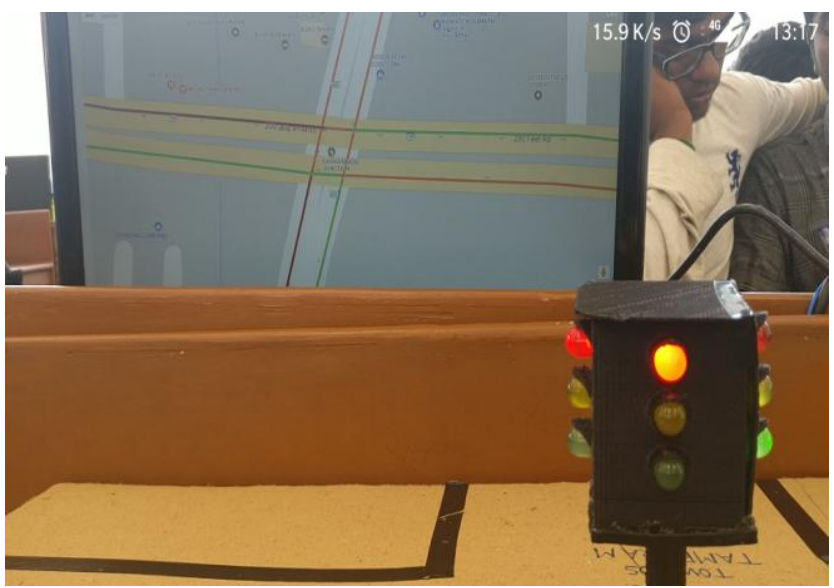

Fig 3 : Initial setup of the Road signals. [All red to start with]

Now when the detection starts, the incoming road with the maximum traffic is detected. This includes detecting the color and also the length of the color. In Fig 3, the road to the right of the signal has the highest vehicular-volume and hence this particular road is given a green for a maximum period of time.

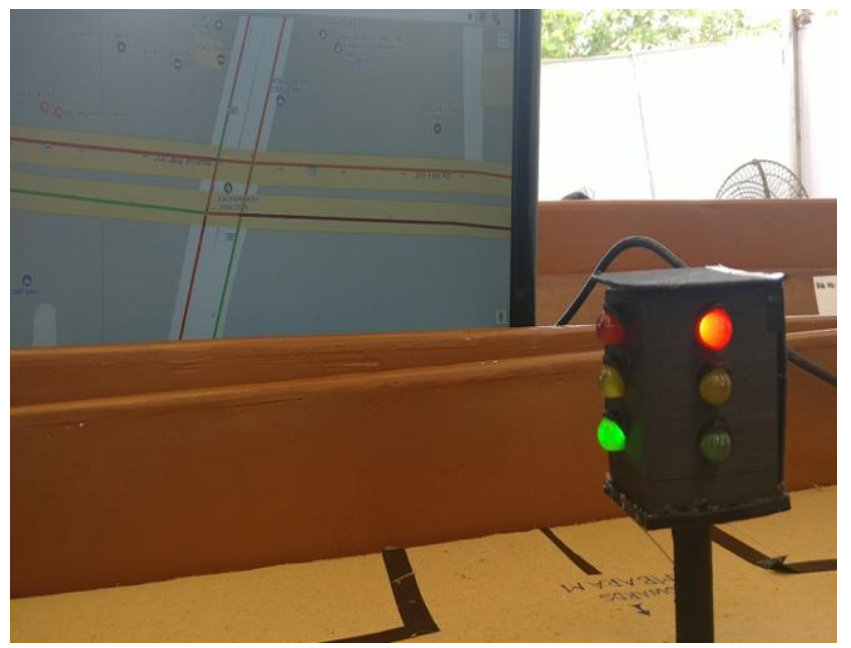

Fig 4 : Another instance of traffic at the road junction

Similarly, the green is transferred to some other road regardless of how these two roads are mutually oriented as given in Fig. 4. There might be two opposite roads or two adjacent roads. So, the overall system doesn't take any conventional (either clockwise or anticlockwise) way of allocating green to roads, instead goes with a tech-driven creative-solution which is the need of an upcoming denselypopulated Indian smart city.

\section{RESULTS AND ANALYSIS Traffic colors}

The color code shows you the speed of traffic on the road.

\section{$\square$ Green: No traffic delays \\ Orange: Medium amount of traffie: \\ Red:Traffic delays. The darker the red, the slower the speed of traffic on the road.}

Note: Gray or blue lines on the map show your routes.

\section{Fig 5 : Google Map Traffic Colors}

Our method classifies roads according to the above mentioned color scheme and gives priority to the road having the least incoming traffic speed.

The proposed method stands out from the traditional and pre-programmed methods and removes equality in green time. This is extremely economical because of the usage of real-time traffic data obtained from Google. In the proposed method the hardware requirements are minimal. Comparatively it is easier to implement the than other solutions and also removes Manual traffic control. Thus traffic has been efficiently optimized for a particular 4-way signal. This model can be extended to work for a 2-way junction as well as a 3-way junction. The whole setup which has been done for one signal can be implemented for all other signals in and around a particular metropolitan city and thus can keep air pollution levels in that city at a check.

\section{CONCLUSIONS}

In this paper we have proposed a method for

Published By:

Blue Eyes Intelligence Engineering \& Sciences Publication

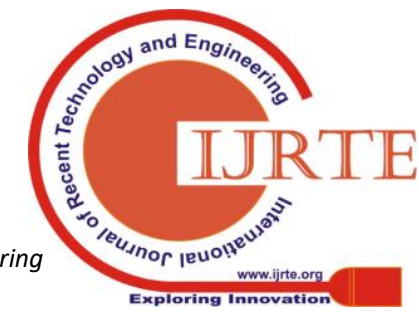


traffic signal optimization using Google traffic data. Our proposed Smart Real-Time Traffic Optimization System (SRTOS) provides optimization of traffic signals based on real time Google traffic data. Every vehicle (having mobile phone users) approaching a traffic-junction contributes to the incoming traffic which is rightfully captured by Google using every mobile phone's GPS locator. These GPS locations are translated to Google map data and are represented through distinct colours. These reliable traffic data providers are used by the proposed Python script to find out incoming traffic through each road of the junction so as to provide traffic-appropriate green times to each segment.

The algorithm developed as a whole can be enhanced by using machine learning for the signal to react to certain abnormal situations. Sometimes during emergency the entire setup may be stopped and manual transmission through traffic control stations may be resorted to. Advanced abnormality-tracking systems can be used in collaboration with the current tech-driven solution to achieve higher levels of perfection in vehicle tracking and movement. Abnormalities include situations like hardware malfunction, adverse weather conditions, traffic deadlock, communication barriers and massive internet failure.

Further, improvements include providing some time for some pedestrian movement such that it coincides with the current implementation and extra time needn't be provided for the same. Some pre-built sensors may also be used to detect any abnormalities (like an incoming ambulance) and this sensor-driven information may be combined with our current traffic information to stop every other process and provide green time for the passing

vehicles on emergency situations and hence such abnormal situations can be tackled efficiently.

\section{REFERENCES:}

[1] Gokulan B.P., Srinivasan D., "Distributed Geometric Fuzzy Multi-agent Urban Traffic Signal Control", IEEE Transactions on Intelligent Transportation Systems, vol.11, no.3, pp.714-727, 2010.

[2] Bilal Ghazal, Khaled EIKhatib, Khaled Chahine, Mohamad Kherfan, " Smart traffic Light Control System ", IEEE 2016.

[3] Abishek C, Mukul Kumar and Kumar Padmanabh "City Traffic Congestion Control in Indian Scenario using Wireless Sensors Network".

[4] Rahul B. Pendor , P. P. Tasgaonkar " An IoT Framework for Intelligent vehicle monitoring System ", International Conference on Communication and Signal www.ijraset.com Volume 5 Issue V, May 2017 IC Value: 45.98 ISSN: 2321-9653 International Journal for Research in Applied Science \& Engineering Technology (IJRASET) CIJRASET: All Rights are Reserved 711 Processing, April 6-8, 2016, India

[5] Ayush Kr. Mittal and Deepika Bhandari, " A Novel Approach to Implement Green Wave system and Detection of Stolen Vehicles", 978-14673-4529-3/12/\$31.00_c 2012 IEEE

[6] Mohammad S. Jassas, Abdullah A. Qasem, Qusay H. Mahmoud, “A Smart System Connecting e-Health Sensors and the Cloud", Proceeding of the IEEE 28th Canadian Conference on Electrical and Computer Engineering Halifax, Canada, May 3-6, 2015.

[7] Srinivasa, R. V., Nageswara, R., \& Kumari, E. K. (2009). Cloud computing: An overview. Journal of Theoretical and Applied Information Technology, 9(1), 71-76.

[8] Buyya, R., Broberg, J., \& Goscinski, A. M. (Eds.). (2010). Cloud computing: Principles and paradigms (Vol. 87). John Wiley \& Sons

\section{AUTHORS PROFILE}

Ramanarayanan $\mathbf{S}^{1 *}$ Student, Computer Science and Engg.,SSN College of Engg. , College, Kalavakkam- 603110, Chennai, Tamil Nadu , India

Udaya Prakash ${ }^{1}$ Student, Information Technology, SSN College of Engg. , College, Kalavakkam-603110, Chennai, Tamil Nadu, India

Uma Maheshwaran Student, Information Technology, SSN College of Engg. , College , Kalavakkam-603110, Chennai, Tamil Nadu, India

Velappa Ganapathy ${ }^{13}$ Professor, SRMIST, Kattankulathur,Chennai603110,Tamilnadu, India

Sundara Kanchana.J ${ }^{14}$ Academic Admininstrator,

SRMIST,Kattankulathur, Chennai-603110,Tamilnadu, India 